\title{
X.
}

\section{Der Gebrauch des Reflexspiegels bei der Untersuchung des Ohres und die Erzeugung vergrösserter Trommelfellbilder}

\author{
von \\ Dr. Trautmann, \\ Oberstabs- tund Regiments-Arzt beim 1. Schles. Grenadier-Regt. Nr. 10 zu Breslat.
}

(Mit 3 Holzschnitten.)

Man benutzt zur Untersuchung des Ohres als Lichtquelle jetzt wohl fast allgemein Tageslicht, und zwar eignet sich am besten dazu ein mit weissen Wolken überzogener Himmel. Bei dieser Beleuchtung treten die grellen Farben roth und gelb nicht so in den Vordergrund, und das Trommelfell erscheint dem Beobachter, wenn auch nicht so intensiv beleuchtet, doch am normalsten in seiner Farbenntiance, worauf grösserer Werth zu legen ist, als auf eine intensive Beleuchtung. Wer im Untersuchen von Ohren geübt ist, bedari zur Beleuchtung des Trommelfells so wenig Licht, dass ihm sogar schon das weisse, bei Sonnenschein herabgelassene Rouleau als Lichtquelle genügt. Die Beleuchtung des Trommelfells mit directem Sonnenlichte oder mit complicirten Beleuchtungs-Apparaten kann wohl nur für den Anfänger etwas Verlockendes haben, weil er meint, er beleuchte sich damit das Trommelfell reeht hell; sobald er aber nur einigermassen getibt ist, wird er das directe Sonnenlicht meiden. Nicht selten kommt der Ohrenarzt in die Lage, Kranke im Bett untersuchen zu müssen. Findet die Untersuchung bei Tage statt, so empfiehlt es sich das Bett an das Fenster rücken zu lassen; ist dies nicht möglich und muss die Untersuchung des Abends gemacht werden, so bediene man sich als licht- 
quelle einfach einer Stearinkerze. Alle complieirten Beleuchtungs-Apparate sind unnöthig. Mit einem Hohlspiegel nun wird das Bild des leuchtenden Gegenstandes (in gewöhnlichen Fällen also das durch Tageslicht erleuchtete Fenster, in aussergewöhnlichen die Flamme der Stearinkerze) durch den in den äusseren Gehörgang gesteckten Trichter auf das Trommelfell reflectirt. Die Verbreitung dieser Untersuchungsmethode verdanken wir hauptsächlich $\vee$. Tröltsch. Alle anderen Untersuchungsmethoden sind als unzureichend und veraltet zu betracnten, weshalb ich sie nicht erwähne, um so weniger, da die Sache schon durch Politzer (Beleuchtungsbilder 1865, S. 13) erledigt ist.

Bei der Untersuchung mit dem Reflexspiegel sind folgende Punkte zu berücksichtigen:

1) die Entfernung der Lichtquelle rom Spiegel,

2) die Entfernung des Spiegelbildes vom Spiegel,

3) die Grösse der Spiegelaxe, resp. des Krümmungshalbmessers.

Diese Punkte lassen sich aus nachstehenden physikalischen Gesetzen erörtern:

a) von einem jenseits des Mittelpunktes der Spiegelaxe befindlichen leuchtenden Gegenstande entsteht zwischen Mittelpunkt und Brennpunkt ein verkehrtes, verkleinertes Bild, das um so grösser, je grösser die Axe des Hohlspiegels ist, d. h. also, welches um so heller, je kleiner die Axe des Hohlspiegels ist;

b) nähert sich der lenchtende Gegenstand dem Mittelpunkte, so nähert sich auch das Bild dem Mittelpunkte;

c) befindet sich der Gegenstand im Mittelpunkte, so entsteht das Bild ebenfalls im Mittelpunkte gleich gross und verkehrt;

d) entfernt sich der leuchtende Gegenstand vom Mittelpunkte, so nähert sich das Bild dem Brennpunkte. Sind leuchtende Gegenstände weiter vom Spiegel entfernt als die 100 fache Länge des Krümmungshalbmessers, so fallen die Bilder ohne merklichen Fehler in den Brennpunkt;

e) befindet sich der Gegenstand zwisehen Mittelpunkt und Brennpunkt, so fällt das Bild über den Mittelpunkt hinaus und ist umgekehrt und vergrössert;

f) je mehr sich der Gegenstand dem Brennpunkte nähert, um so grösser und weiter entfernt ist das Bild vom Mittelpunkte;

g) von einem zwischen dem Brennpunkte und Spiegel ge- 
legenen leuchtenden Gegenstande entsteht ein aufrechtes vergrössertes Bild hinter dem Spiegel.

Die von $a$ bis $f$ entstehenden Bilder, bei denen die reflectirten Strahlen sich selbst durchschneiden, nennt man physische oder Sammelbilder; das Bild von diesen lässt sich auf einem Schirm auffangen. Im Gegensatz zu den physischen Bildern haben wir die virtuellen oder geometrischen, die entstehen, wenn sich die auffallenden Strahlen in ibren Verlängerungen schneiden. Ein solches Bild entsteht sub $g$, ist aufrecht und vergrössert hinter dem Spiegel zu sehen und eignet sich zur Trommelfelluntersuchung nicht, da es nicht aufzufangen ist.

Von den angeführten Bildern eignet sich das sub $a$ angefuhrte am besten zur Trommelfelluntersuchung, weil es am intensirsten ist und die Entfernung der Lichtquelle rom Spiegel bei der Untersuchung des Trommelfelles am zweckmässigsten so gewählt wird, dass sie jenseits des Mittelpunktes der Spielaxe liegt.

Bei der Untersuchung stelle man den Kranken in die Nähe des Fensters, das zu untersuchende $\mathrm{Ohr}$ abgewandt von demselben, so dass dasselbe etwa 1 Fuss rom Fenster entfernt ist, mit dem 3. und 4. Finger zieht man die Ohrmuschel nach hinten und oben, mit dem Zeigefinger und Daumen stellt man sich den in den äusseren Gehörgang gesteckten Trichter passend ein. Mehrfach ist empfohlen mit Mittel- und Zeigefinger die Obrmuschel nach hinten und oben zu ziehen (v. Tröltsch, Ohrenheilk. 1868, S. 59, Politzer, Beleuchtungsbilder S, 16) und mit dem Daumen den unteren Rand des Trichters zu stützen. Ich halte die vorerwähnte Methode, welche Schwartze lehrt, für besser, weil man mit Zeigefinger und Daumen den Trichter rotirend bewegen kann und so keinen Schmerz erzeugt, was bei Druck mit dem Daumen allein leicht geschieht. Die bequemste Entfernung des Reflexspiegels von dem äusseren Gehörgange beträgt etwa $5 \mathrm{Zoll}$; nimmt man nun den äusseren Gehürgang $24 \mathrm{Mm}$., also etwa 1 Zoll nach v. Tröltseh dazu, so beträgt die Entfernung des auf das Trommelfell reflectirten Spiegelbildes $5+1=6$ Zoll.

Ist nun $a$ (Fig. 1) ein leuchtender Punkt in der Spiegelaxe; $a^{\prime}$ das Bild desselben, so ist $L_{-} a G C=L^{\prime} a^{\prime} G C$ und es verhält sich demnach

$$
\text { a } G: a^{\prime} G=a C: a^{\prime} C .
$$


Ist die Weite des Spiegels, also auch der Bogen $D G$ klein, so kann man ohne wesentlichen Fehler $a G=a D$ und

Fig. 1.

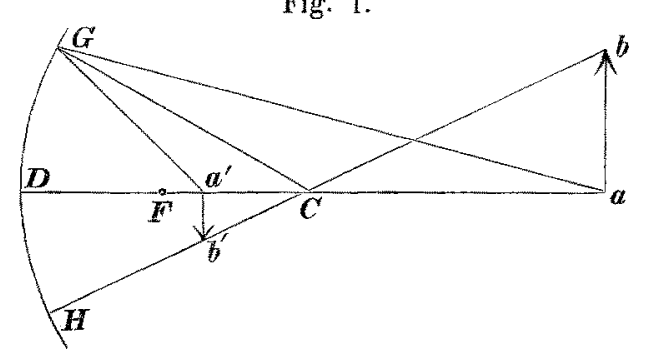

$a^{\prime} G=a^{\prime} D$ setzen, so dass $a D: a^{\prime} D=a C: a^{\prime} C$. Nennt man nun die Entfernung des leuchtenden Gegenstandes vom Reflexspiegel $a D$ $=a$, die Entfernung: des Spiegelbildes rom Reflexspiegel $a^{\prime} D=\alpha$

und die Brennweite des Hoblspiegels $F D=F C=F$, also $C D=2 F$ so verhält sich:

$$
\begin{aligned}
& a: \alpha=a-2 F: 2 F-\alpha \\
& \text { also } 2 a F-a \alpha=a \alpha-2 \alpha F \\
& 2 a F+2 \alpha F=2 a \alpha \\
& a F+\alpha F=a \alpha .
\end{aligned}
$$

Dividirt man nun dureh a $F \alpha$, so erhält man die einfache Gleichung

$$
\frac{1}{a}+\frac{1}{a}=\frac{1}{F}
$$

Man kann nun, wenn zwei Grössen bekannt sind, die dritte leicht berechnen. In unserem oben erwähnten 1. Falle nun beträgt:

$$
\begin{aligned}
& a=5+1=6 \text { Zoll } \\
& a=12+5=17 \text { Zoll. }
\end{aligned}
$$

Man kann also leicht ausrechnen, wie gross die Brennweite des Hohlspiegels in diesem Falle sein muss.

$$
\begin{aligned}
& \frac{1}{6}+\frac{1}{17}=\frac{1}{F} \\
& \frac{23}{102}=\frac{1}{F} \\
& 23 F=102 \\
& F=4,44 .
\end{aligned}
$$

Der Reflexspiegel muss demnach in dem eben besprochenen 1. Falle eine Brennweite von etwa 4,5 Zoll haben.

Benutzt man 2. als leuchtenden Gegenstand die Wolken oder eine gegenüberliegende belle Wand, also Gegenstände, welche weiter vom Spiegel entfernt sind, als die 100 fache Länge 
des Krümmungshalbmessers desselben, so fällt das Spiegelbild (sub $d$ ) ohne merklichen Fehler in den Brennpunkt. Bedient man sich also in diesem 2. Falle eines Reflexspiegels von der im 1. Falle angegebenen Brénnweite 4,5 Zoll, so müsste man, um das Spiegelbild genau auf das Trommelfell zu reflectiren, 4,5-1 Zoll, also 3,5 Zoll mit dem Spiegel vom äusseren Gehörgang entfernt sein. - Will man nun den Reflexspiegel 5 Zoll vom äusseren Gehörgang entfernt halten, wie im 1. Falle, so kann man $F$ leicht ausreehnen nach obiger Gleichung:

$$
\begin{gathered}
a=\infty, \alpha=5+1=6 \\
\text { also: } \frac{1}{\infty}+\frac{1}{6}=\frac{1}{F} \\
\frac{1}{6}=\frac{1}{F}-\frac{1}{\infty} \\
\frac{1}{6}=\frac{1}{F} \\
F=6 .
\end{gathered}
$$

In dem 2. Falle, wo die Lichtquelle unendlich weit entfernt ist, müsste also der Reflexspiegel eine Brennweite von 6 Zoll haben, wenn ich mit demselben 5 Zoll vom äusseren Gehörgang. entfernt sein will. Es bedarf wohl kaum der Erwähnung, dass wer sich verleiten lassen sollte pit directem Sonnenlichte zu untersuchen, nicht noch den Fehler begehen darf, den Reflexspiegel so zu halten, dass dessen Brennpunkt auf die Ohrmuschel, den äusseren Gehörgang oder das Trommelfell fällt, da in diesem Falle der Hohlspiegel als Brennspiegel wirkt. In vielen Fallen ist es wünschenswerth, die rechte Hand zum Operiren frei zu haben, und ist es in diesen Fällen empfohlen, den Spiegel durch ein Brillengestell zu befestigen. Wenn ich auch diese Methode nicht fur unpraktisch halte, so macht es doch auf mich einen ganz eigenthümlichen Eindruck, wenn ich einen Arzt sehe, der sich das rechte Auge mit einem Reflexspiegel verbunden hat; dass der Eindruck auf die Kranken gewiss auch nicht angenehm ist, möchte ich ebenfalls behaupten. Schwartze hält den Reflexspiegel in den Fällen, wo er die rechte Hand frei haben will, mit dem Zeigefinger und Daumen der linken Hand, mit dem 3. Finger lenkt er den Trichter und mit dem 4. und 5. Finger hält er die Ohrmuschel. Ich will nicht in Abrede stellen, dass diese Methode, wo eine Hand drei Verrichtungen machen soll, für den Anfänger manche Schwierigkeit hat, geübt aber bald erlernt wird und jedenfalls keinen unan- 
genehmen Eindruck macht. Zur Erleichterung der Handhabung habe ich mir am Spiegelgriffe einen verstellbaren Ring für den linken Daumen anbringen lassen, ähnlich dem am Ende der Wilde'schen Schlinge; man kann dann bequem den Spiegel halten und 5 Zoll von der Ohrmuschel entfernen, so dass derselbe Reflexspiegel ron 4,5 Zoll Brennweite wie im 1. Falle auch im 3. Falle geniugt. v. Tröl ts e h (a.a. 0.S.62) empfiehlt Reflexspiegel von 5-6 Zoll Brennweite. Ist $F=5$, so muss ich im 1 . Falle, wenn die Lichtquelle 17 Zoll entfernt ist, 6 Zoll vom äusseren Gehörgange entfernt sein mit dem Spiegel, oder wenn ich 5 Zoll entfernt sein will, muss die Lichtquelle 30 Zoll vom Reflexspiegel entfernt sein. Im 2. Falle wenn der leuchtende Gegenstand unendlich. weit, und $F=5$ ist, muss ich 4 Zoll vom äusseren Gehörgang entfernt sein. Im 3. Falle müsste ich den Reflexspiegel auf 6 Zoll entfernt halten, was mit der linken Hand allein schwer gelingen würde, deshalb muss ich die Lichtquelle auf 30 Zoll entfernen, oder auf unendlich und dann mit dem Spiegel auf 4 Zoll an den äusseren Gehörgang gehen. Ist $F=6$ Zoll, so muss ich im 1. Falle 8 Zoll rom äusseren Gehörgang entfernt sein mit dem Spiegel, oder wenn ich 5 Zoll entfernt sein will, muss die Lichtquelle unendlich weit entfernt sein oder wenigstens über dass 100 fache des Krümmungshalbmessers, also itber 50 Fuss.

Im 2. Falle passt der Brennpunkt des Spiegels, ich kann an die Ohrmuschel auf 5 Zoll herangehen, wenn die Liehtquelle unendlich weit entfernt ist. - Im 3. Fall kann ich mit der linken Hand nicht 8 Zoll den Spiegel mit dem Daumen der linken Hand entiernen, ich muss also die Lichtquelle von unendlich weit nehmen.

Man sieht also, dass sich Reflexspiegel von 5-6 Zoll Brennweite sehr gut verwerthen lassen, wenn man die Entfernung der Lichtquelle dazu anpasst.

Politzer (Beleuchtungsbilder S. 15) nimmt den Spielraum des Brennpunktes noch weiter, 4-6 Zoll; und sagt (Seite 17): "der Spiegel wird dem $\mathrm{Ohr}$ in so weit genähert, dass die grösste Intensität des Lichtes das Trommelfell erreicht (Focaldistanz)." Das Wort "Focaldistanz" wäre besser fortgelassen; denn sollte es aufrecht erhalten werden, so miisste die Lichtquelle stets unendlich weit eingenommen werden, und die Entfernung wäre vom äusseren Gehörgange bei einem Reflexspiegel, dessen Focaldistanz 4-6 Zoll wäre, 3-5 Zoll; und 3 Zoll dürfte etwas zu 
nahe sein. Nimmt man die Lichtquelle nieht als unendlich an, so müsste sie bei einer Focaldistanz des Spiegels von $4 \mathrm{Zoll}$, wenn man denselben 5 Zoll von dem äusseren Gehörgang entfernt halten will, 12 Zoll vom Spiegel entfernt sein. So lassen sich auch die ubrigen Entfernungen nach obiger Gleichung leicht ausrechnen, was ich jedoch unterlasse. Bei den jetzt käuflichen Reflexspiegeln hat man meist eine Focaldistanz von 8 Zoll, so dass man, wenn die Entfernung der Lichtquelle unendlich weit, 7 Zoll vom äusseren Gehörgang entfernen müsste. Im 3. Falle ist der Spiegel gar nicht zu verwerthen. Diese Reflexspiegel mit 8 Zoll Brennweite empfehlen sich also nicht; jedenfalls hat man sie für unendlich weite Lichtquellen berechnet.

Die Grössendurchmesser der Reflexspiegel sind auch verschieden angegeben, v. Tröltseh (Ohrenheilkunde S. 62) nicht unter $23 / 4-3$ Zoll bei 5-6 Zoll Brennweite, Politzer (Beleuchtungsbilder S. 15) $4-5$ Zoll bei $4-6$ Zoll Brennweite.

Die Strahlen von leuchtenden Gegenständen gehen divergent aus und werden convergent vom Reflexspiegel in den Trichter geworfen. Es können also nur soviel convergente Strahlen aufgenommen werden, als die Breite des Trichtereinganges aufzunebmen im Stande ist. Die Weite des äusseren Gehörganges ist zu verschieden, als dass man danach das eindringende Strahlenbündel berechnen könnte. Man benutzt, um dem Lichte besseren Eingang zu verschaffen, kegelförmige Trichter, deren Oeffnung nach aussen $13-15 \mathrm{Mm}$. beträgt. Der Trichter dringt meist nur so weit in den äusseren Gehörgang, dass er noeh $1 / 2-3 / 4$ Zoll aus demselben heraussieht. - Nehme ich nun die Weite der äusseren Trichteröffnung Fig. 2 a $b \begin{array}{lll} & 13\end{array}$

Fig. 2. 1/2 der natïrlichen Grösse.

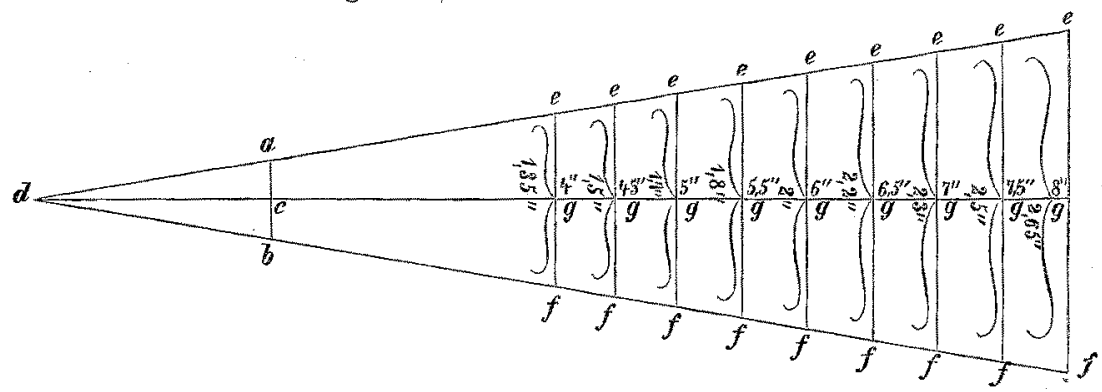


bis $15 \mathrm{Mm}$. und errichte darauf eine Senkrechte c d $11 / 2$ Zoll lang (Länge des Gehörganges + dem heraussehenden Trichterstïck), verlängere diese nach $g$ und ziehe durch die Punkte $a$ und $b$ die Linie $d e$ und $d f$, so liegt in dem Strahlenbündel $e$ $d f$ der Grössendurchmesser für den Reflexspiegel, dessen Breite für die entsprechende Brennweite auf der Linie $d g$ abzumessen ist.

Hiernach ergeben sich durch Construction folgende Werthe: ist die Trichteröffnung $13 \mathrm{Mm}$. weit und $1 \frac{1}{2}$ Zoll vom Trommelfell entfernt, so ist bei einem Reflexspiegel

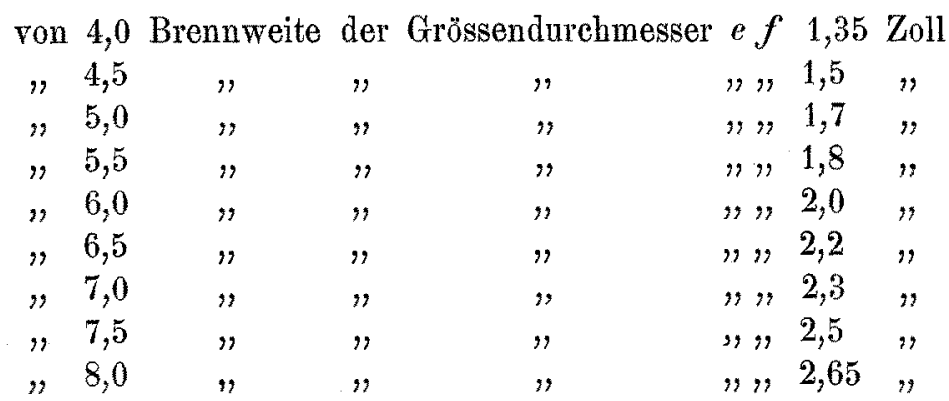

Ist die Trichteröffnung $15 \mathrm{Mm}$. und dieselbe $1 \frac{1 / 2}{2}$ Zoll vom Trommelfell entfernt, so ändert sich der Grössendurchmesser sehr wenig, da der $L_{-} a d b$ nur um 10 Minuten geändert wird. Ich unterlasse es deshalb für diese Weite die Werthe anzuführen.

Man kann die Grössendurehmesser auch noch auf andere Weise finden:

1) durch das Verhältniss (Fig. 2) $e f: a b=d g: d c$.

$$
\begin{aligned}
e f & =\frac{a b \cdot d g}{d c} \\
a b & =13 \mathrm{Mm} .=1 / 2 \text { Zoll } \\
d g & =\text { Brennweite } \\
d c & =1,5 \text { Zoll } \\
\text { also } e f & =\frac{0,5 \cdot 4}{1,5} \\
e f^{\prime} & =1,33 \text { bei } 4^{\prime \prime} \text { Brennweite; }
\end{aligned}
$$

2) durch Trigonometrie. Man hat dann gleichzeitig eine Controlle, ob die durch Construction gefundenen Werthe richtig sind.

Nach Fig. 2 ist $e g=g d$ tang $e d g ; g d=$ Brennweite des Spiegels $e d g=10^{\circ}$ 
hiernach erhält man folgende Werthe:

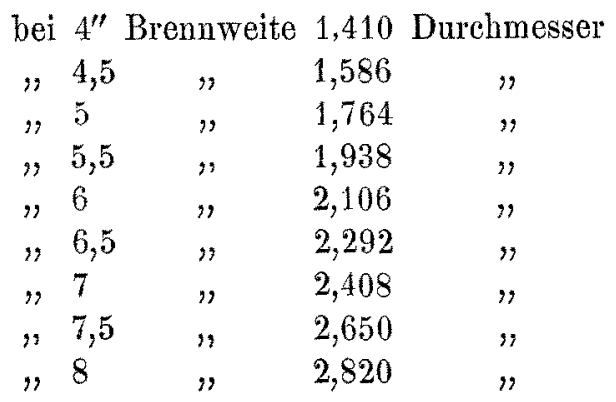

Wir sehen also, dass die durch Construction gefundenen Werthe wenig von den ausgerechneten abweichen. Die Grössendurchmesser der Reflexspiegel sind hiernach ziemlich klein.

Da nun aber die Randstrahlen nicht so intensiv sind, als centrale, ferner selbst beim besten Spiegel böchstens $2 / 3$ der auffallenden Strablen reflectirt werden und ausserdem bei der Untersuchung mit dem Spiegel seitliche Bewegungen gemacht werden, so nimmt man zweckmässig den Durchmesser 1 Zoll grösser; diese Grösse genügt vollständig; und die von Politzer angegebenen Durehmesser sind zu hoch gegriffen; es würde bei Spiegeln von 4-6 Zoll Brennweite ein Durchmesser von 2,41 bis 3,10 Zoll vollständig genügen. - Die von $\nabla$. Tröltsch angegebenen Durchmesser $2^{3 / 4}-3$ Zoll für Brennweite von 5-6 Zoll sind vollkommen zweckentsprechend und stimmen ganz genau mit obiger Berechnung, wenn 1 Zoll addirt wird.

Da nun, wie ich gleich im Eingange bemerkt, der Geübte zur Untersuchung des Trommelfells sehr wenig Licht gebraucht, so ist es nicht nothwendig, die so eben angefuhrten Entfernungen ganz genau fest zu halten. Der Geübte wird auch mit Spiegeln von der rerschiedensten Brennweite untersuchen können, weil er eben nicht nöthig hat, das Bild so auf das Trommelfell zu reflectiren, dass es am intensivsten erleuchtet ist. Die bequemsten für alle Fälle passenden Spiegel, wie ich dies auch praktisch ausprobirt habe, sind Concarspiegel mit 5 Zoll Brennweite und 3 Zoll Durchmesser; man hat dabei vielleicht nur in manchen Fällen nöthig die Entfernung der Lichtquelle zu reguliren, was nach obiger Gleichung leicht zu berechnen ist. 
Vergrosserte Trommelfellbilder.

Für sphärische Gläser oder Linsen, d. h. für Gläser, deren Flächen Kugelabschnitte sind, gelten dieselben Gesetze wie für spbärische Spiegel. Die oben entwickelte Gleichung $\frac{1}{a}+\frac{1}{a}=\frac{1}{F}$ ist also auch hier maassgebend. Wenn sich nun ein leuchtender Gegenstand innerhalb der Brennweite der Linse befindet, so entsteht (siehe oben sub $g$ ) von demselben kein physisehes, sondern ein geometrisches, aufrechtes und vergrössertes Bild, welches von der linse weiter entfernt ist als der Gegenstand. Dieses Bild riłkkt um so weiter hinaus und ist folglich um so grösser; je näher der Gegenstand sich dem Brenupunkte befindet. Will ich nun vergrösserte Trommelfellbilder erzengen, so muss ich eine biconvexe Linse zwischen Trommelfell und Reflexspiegel einschieben; und zwar muss das Trommelfell so weit entfernt von der Linse sein, dass es innerhalb der Brennweite derselben liegt. - Halte ich also die Linse dicht an die äussere Trichteröffnung, so muss dieselbe, da die Trichteröffnung meist $1 / 2-3 / 4$ Zoll den äusseren Gehörgang überragt, also etwa $13 / 4$ Zoll vom Trommelfell entfernt ist, eine grössere Brennweite als 13/4 Zoll haben. Linsen von $2^{1 / 4}-3^{1 / 4}$ Zoll Brennweite eignen sich am besten. Die Bilder sind bei Linsen mit 21/2 Zoll Brennweite am schönsten, wenn auch nicht am grössten.

Ich habe, um dieses Untersuchungsverfahren so einfach als möglich und gar nicht abweichend von dem früheren zu gestalten, Triehter anfertigen lassen, zu denen 3 Linsen mit entsprechender Brennweite gehören, die in die Oeffnung der Trichter eingeschraubt werden können, so dass ich beliebig Triehter und Linsen wechseln kann.**) Die Oeffnung des Trichters ist etwas abgeschrägt, wodurch die Linse schräg gestellt wird, damit das Spiegelbild des Reflexspiegels nicht auf der Linse stört. Es gehört beinahe gar keine Uebung dazu, um sich sofort vom Trommelfell ein vorzügliches vergrössertes Bild zu verschaffen. Man sieht Flächenuntersehiede, Verkalkungen, Trübungen, Abweichungen in der Form des Lichtreflexes, Gefässerweiterungen etc. so vorzüglich und gewinnt î̉ber manche Sachen einen so ausgezeichneten Einblick, dass ich glaube, man wird diese Trichter mit den Convexlinsen vielfach in Gebrauch ziehen. Die

*) Instrumentenmacher Pischel, Weidenstr, 5 in Breslar, liefert diese Trichter. 
kegelförmigen Trichter eignen sich am besten dazu, da diese ein grösseres Strahlenbïndel aufnehmen und durch die Linse gehen Jassen als die cylindrischen, die an der äusseren Oeffnung ausgeschweift sind; durch letztere geht nur das Strahlenbündel, welches die Weite des Cylinders aufnehmen kann.

Betrachten wir nun jetzt ein Trommelfell mit einer Linse von 21/4 Zoll Brennweite.

Nach obiger Gleichung ist: $\frac{1}{a}+\frac{1}{\alpha}=\frac{1}{F}$

$a$ die Entfernung des Trommelfells von der Linse $=1+3 / 4$ Zoll

$F=$ dem Brennpunkte der Linse $=2,25$ Zoll

$=1,75 \mathrm{Zoll}$

$\alpha$ die Entfernung des vergrösserten Bildes,

$$
\begin{aligned}
& \text { also: } \frac{1}{1,75}+\frac{1}{\alpha}=\frac{1}{2,25} \\
& \frac{2,25}{1,75}+\frac{2,25}{\alpha}=1 \\
& \frac{2,25}{1,75} \alpha+2,25=\alpha \\
& 2,25 \alpha+3,93=1,75 \alpha \\
& 0,5 \quad \alpha=-3,93 \\
&-\alpha=-7,8 ;
\end{aligned}
$$

das vergrösserte Bild ist also 7,8 Zoll von der Linse entfernt. Der. Werth ist negativ, da Object und vergrössortes Bild nach derselben Seite liegen.

Hat die Linse eine Brennweite

von 2,5 Zoll, so ist das Bild 5,8 Zoll von der Linse entfernt

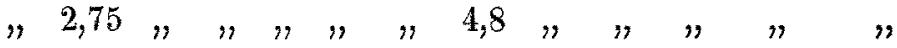

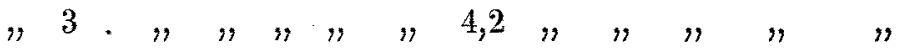

$$
\begin{aligned}
& \text { "3,25 " " " " } " 3,8 \text { " " " " " }
\end{aligned}
$$

Ist nun (Fig. 3) $A B$ eine Linse von 2,25 Zoll Brennweite,

Fig. 3.

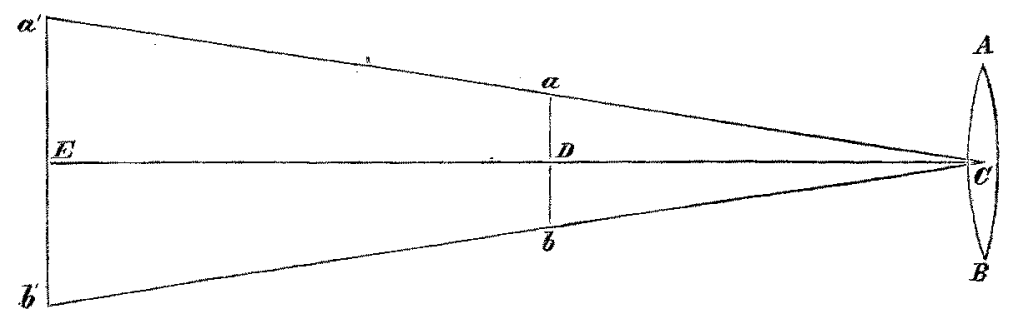


$C D$ die Entfernung des Trommelfells von der Linse $=1,75 \mathrm{Zoll}$, $a b$ der Durchmesser des Trommelfells $=9 \mathrm{Mm}$. ca. $=1 / 3$ Zoll (Strieker S.842), so kann ich nach obiger Gleichung $\frac{1}{a}+\frac{1}{\alpha}=\frac{1}{\bar{F}}$ die Entfernung $C E$ ausrechnen und sind die Werthe vorher soeben angegeben; darnach ist $C E$ für die Brennweite von 2,25 Zoll $=7,8 \mathrm{Zoll}$.

Nun verhält sich: $a b: a^{\prime} b^{\prime}=C D: C E$

$$
\begin{aligned}
& 1 / 3: a^{\prime} b^{\prime}=1,75: 7,8 \\
& 1,75 a^{\prime} b^{\prime}=2,6 \\
& a^{\prime} b^{\prime}=1,48 .
\end{aligned}
$$

Die lineäre Vergrösserung des Trommelfells beträgt also für eine Linse von: $2,25 \mathrm{Zoll}=1,48$ Zoll also 4,44 Mal Auf diese Weise lassen sich die Vergrösserungen für die iibrigen Linsen berechnen und sind

$$
\begin{aligned}
& 2,5 \text { Zoll }=1,08 \text { Zoll also } 3,24 \text { Mal } \\
& 2,75 \quad "=0,91 \quad " \quad " \quad 2,73 \quad " \\
& 3,0 \quad "=0,8 \quad " \quad " \quad 2,4 \quad " \\
& 3,25 \quad "=0,72 \quad " \quad " 1,44 \quad "
\end{aligned}
$$

Je stärker nun das Bild vergrössert wird, um so mehr nimmt die Lichtstärke desselben, und zwar im quadratischen Verhältnisse der Vergrösserung $a b$; ausserdem wachsen mit der Vergrösserung die Fehler der sphärischen und chromatischen Abweichung, so dass es sich empfiehlt, Linsen zu nehmen, wo das Object nicht zu nahe an den Brennpunkt fällt. Am besten habe ich die linse mit einer Brennweite von $21 / 2$ Zoll gefunden.

Was nun die Brennweite des Reflexspiegels und die Entfernung der Lichtquelle betrifft, so ändern sich auch diese, da das vergrösserte Bild weiter hinaus gerückt wird. Die Brennweite der Reflexspiegel muss hier um so mehr beachtet werden, weil mit der Vergrősserung die Lichtstärke im quadratischen Verhältniss abnimmt. Bei einer linse nun von 2,25 Zoll Brennweite ist die Entfernung des Bildes von der Linse = 7,8 Zoll; die Entfernung der Linse bis zum äusseren Gehörgang beträgt 0,75 Zoll, den Spiegel balte ich am bequemsten 5 Zoll vom äusseren Gehörgang entfernt, also beträgt die Entfernung des vergrö̀sserten Bildès vom Reflexspiegel $7,8-0,75+5$. Will 
ìch nun die Brennweite des zu benutzenden Reflexspiegels wissen, wenn die Lichtquelle 17 Zoll entfernt ist, so ist:

$$
\begin{aligned}
& \frac{1}{17}+\frac{1}{7,8-0,7+5}=\frac{1}{F} \\
& \frac{1}{17}+\frac{1}{12,1}=\frac{1}{F} \\
& \frac{12,1}{17}+1=\frac{12,1}{F} \\
& 12,1+17=\frac{205,7}{F} \\
& 29,1 F=205,7 \\
& F=7,0 .
\end{aligned}
$$

Will ich jedoch den oben empfohlenen Reflexspiegel von 5 Zoll beibehalten, so muss die Entfernung der Lichtquelle verändert werden. Es wird dies Jeder beim Untersuchen mit Linsen sofort selbst merken, da er nur in dem Fall klare, vergrösserte Trommelfellbilder erhält, wenn diese Verhältnisse richtig gewählt werden. Um nun die Entfernung der Lichtquellen zu berechnen, ist

$$
\begin{gathered}
\frac{1}{a}+\frac{1}{12,1}=\frac{1}{5} \\
1+\frac{a}{12,1}=\frac{a}{5} \\
12,1+a=\frac{12,1 a}{5} \\
60,5+5 a=12,1 a \\
60,5=7,1 a \\
a=8,5 .
\end{gathered}
$$

Ich muss also die Lichtquelle auf 8,5 Zoll nähern. Diese Verhältnisse treten bei der Untersuchung mit einer Stearinkerze sehr deutlich hervor. Die Werthe nun für die ibrigen Linsen sind folgende:

Bei einer Linse von

$2,5^{4}$ Brennweite muss die Brennweite des Reflexspiegels $6,3^{\prime \prime}$ betragen bei

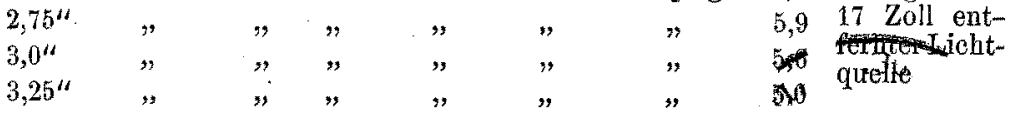

Bei einer Linse von

2,5" Brennweite muss die Lichtquelle 9,9 Zoll entfert seim, menn der Re-

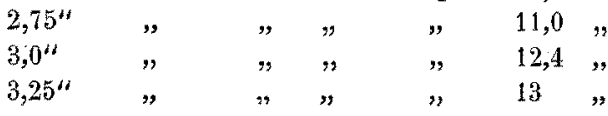
flexsongyel Oexine Bntengweita von thil hat 
Wir sehen also, dass ein Reflexspiegel von 5 Zoll Brennweite auch für vergrösserte Bilder passt, wenn man nicht zu starke Vergrösserung wählt. - Zweckmässiger wählt man aber zu diesem Zwecke Concavspiegel mit grösseren Brennweiten, wozu die soeben angegebenen Brennweiten Anhaltspunkte geben. Ich benutze den jetzt gewöhnlich käuflichen mit 8 Zoll Brennweite für vergrösserte Trommelfellbilder, und muss dann die Lichtquelle

bei einer Linse von 2,25 Brennweite $=21$ Zoll entfernt sein

\begin{tabular}{|c|c|c|c|c|c|c|c|c|c|}
\hline$"$ & $n$ & $" 7$ & $"$ & 2,5 & $"$ & $=38,3$ & $"$ & $"$ & $"$ \\
\hline$"$ & $" 1$ & $n$ & $n$ & 2,75 & $"$ & $=66$ & $"$ & $"$ & $"$ \\
\hline$n$ & $"$ & $n$ & $"$ & 3,0 & $"$ & $=136$ & $"$ & $n$ & $" 1$ \\
\hline$\eta$ & $n$ & $n$ & $n$ & 3,25 & $n$ & $=648$ & "y & 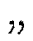 & $"$ \\
\hline
\end{tabular}

